PROCEEDINGS OF THE

AMERICAN MATHEMATICAL SOCIETY

Volume 131, Number 9, Pages 2705-2710

S 0002-9939(03)06817-5

Article electronically published on January 15, 2003

\title{
CANCELLATION OF DIRECT SUMS OF COUNTABLE ABELIAN $p$-GROUPS
}

\author{
RÜDIGER GÖBEL AND WARREN MAY
}

(Communicated by Stephen D. Smith)

\begin{abstract}
Let $B \oplus A_{1}=C \oplus A_{2}$ be abelian groups where $B \cong C$ is a direct sum of countable $p$-groups. A condition is given on the Ulm-Kaplansky $p$ invariants of $B, A_{1}$ and $A_{2}$ such that $A_{1} \cong A_{2}$.
\end{abstract}

Let $p$ denote a fixed prime number. In [3], the following result is shown for two isomorphic modular abelian group algebras: if one group is an $\aleph_{1}$-separable abelian $p$-group of cardinality $\aleph_{1}$, then the two groups are isomorphic under the assumption of MA and $\neg \mathrm{CH}$. A question which arises in the proof is a variation of the "substitution property" in Problem 58 in 2, namely, can a direct sum of cyclic $p$-groups be cancelled from isomorphic direct sums if the Ulm-Kaplansky invariants of the direct sum of cyclics are "disjoint" from those of the complementary groups. In [1], Crawley proved a cancellation theorem for totally projective groups with all Ulm-Kaplansky invariants finite. Such groups are of necessity countable. We shall prove a cancellation theorem which has both Crawley's theorem and a positive answer to the question above as corollaries. Specifically, we prove the

Theorem. Let $G=B \oplus A_{1}=C \oplus A_{2}$, where $B \cong C$ is a direct sum of countable abelian p-primary groups, and $A_{1}$ and $A_{2}$ are arbitrary abelian groups. For every Ulm-Kaplansky p-invariant of $B$, assume that it is either finite or else the corresponding Ulm-Kaplansky invariants of $A_{1}$ and $A_{2}$ are zero. Then there exists a subgroup $D$ of $G$ such that $G=D \oplus A_{1}=D \oplus A_{2}$. In particular, $A_{1}$ and $A_{2}$ are isomorphic.

Conjecture. The Theorem is true if $B$ is allowed to be a totally projective p-primary group.

In fact, we know of no counterexample if $B$ is allowed to be an arbitrary $p$-group.

All Ulm invariants will be understood to be Ulm-Kaplansky invariants for the prime $p$. We recall the definition. For an ordinal $\alpha$, the $p$-socle elements of $p$-height $\geq \alpha$ form a vector space over the integers modulo $p$. The dimension of the quotient space modulo the subspace of elements of $p$-height $>\alpha$ is the Ulm invariant at $\alpha$. The Ulm invariant at $\infty$ is the dimension of the $p$-socle of the maximal divisible $p$-subgroup. It will be useful to be able to assume that $B$ is a reduced $p$-group, so we prove a brief lemma to that effect.

Lemma 1. To prove the Theorem, we may assume that $B$ is reduced.

Received by the editors January 25, 2002 and, in revised form, April 13, 2002.

2000 Mathematics Subject Classification. Primary 20K10, 20K21, $20 \mathrm{~K} 25$.

(C)2003 American Mathematical Society 
Proof. Assume the hypothesis of the Theorem and first suppose that the Theorem is true if $B$ is reduced or divisible. For arbitrary $B$, we may choose a reduced complement $B^{\prime}$ (respectively, $C^{\prime}$ ) for the maximal divisible subgroup of $B$ (respectively, $C$ ). Then the Theorem can be applied to $B^{\prime}$ to obtain $D^{\prime}$ such that $G=D^{\prime} \oplus B^{\prime} \oplus A_{1}=D^{\prime} \oplus C^{\prime} \oplus A_{2}$. Passing to $G / D^{\prime}$, applying the reduced case of the Theorem, and taking inverse image, one obtains $D \supseteq D^{\prime}$ such that $G=D \oplus A_{1}=D \oplus A_{2}$. Thus the lemma will be shown if we prove the Theorem for the case that $B$ is divisible.

Assume now that $B$ is divisible. If the Ulm invariant of $B$ at $\infty$ is infinite, then $A_{1}$ and $A_{2}$ are reduced, hence $B=C$ and we may take $D=B$. If the Ulm invariant of $B$ is finite, then by induction and grouping appropriate summands of $B$ and $C$ with $A_{1}$ and $A_{2}$, we may assume that $B \cong \mathbb{Z}\left(p^{\infty}\right)$. Let $b$ and $c$ be generators for the socles of $B$ and $C$, respectively. Then $b=c^{\prime}+a_{2}$ for some $c^{\prime} \in C, a_{2} \in A_{2}$. If $c^{\prime}$ has order $p$, then $G=B \oplus A_{2}$, thus we may take $D=B$. Therefore we may assume that $b=a_{2}$ and, by symmetry, $c=a_{1}$ for some $a_{1} \in A_{1}$. We may choose $D \cong \mathbb{Z}\left(p^{\infty}\right)$ with socle generated by $b+a_{1}$. Since $b+a_{1}=c+a_{2}$, we have $G=D \oplus A_{1}=D \oplus A_{2}$, as desired.

The proof will need several lemmas, first treating the bounded case and then the countable case. In Lemma 5 we shall use Crawley's idea of induction on the Ulm length, which will be feasible since we may assume that $B$ is reduced. We say that two groups have disjoint Ulm invariants if corresponding Ulm invariants are never both nonzero.

Lemma 2. Let $G=B \oplus B^{\prime} \oplus A_{1}=C \oplus C^{\prime} \oplus A_{2}, C \subseteq B \oplus A_{1}$, and let $\pi: G \rightarrow C$ be the projection with kernel $C^{\prime} \oplus A_{2}$. Assume that $C$ is a p-group and that $C$ and $A_{1}$ have disjoint Ulm invariants. Then $C / \pi(B)$ is divisible. In particular, if $C$ is bounded, then $C=\pi(B)$ and we can conclude that $G=B+\left(C^{\prime} \oplus A_{2}\right)$.

Proof. It will suffice to show that $C \subseteq \pi(B)+p C$. Let $c \in C$ and write $c=$ $b+a\left(b \in B, a \in A_{1}\right)$. Denote the $p$-height of an element $g \in G$ by $|g|$. We first claim that if $c$ has order $p$, then $|c|<|a|$. We may assume that $a \neq 0$, thus $a$ has order $p$ and $|c| \leq|a|$. If $|c|=|a|$, this would contradict the assumption on Ulm invariants, thus the claim is shown. Let the order of $c$ be $p^{k}, k \geq 1$. We will show by induction on $k$ that $c \in \pi(B)+p C$. We have $c=\pi(b)+\pi(a)$, so we must show that $\pi(a) \in \pi(B)+p C$. If $k=1$, then by our claim, $|a| \geq 1$, hence $\pi(a) \in p C$. Now assume $k>1$. The order of $a$ is $\leq p^{k}$, so by induction we may assume it is $p^{k}$. Consider $p^{k-1} c=p^{k-1} b+p^{k-1} a$. Applying the claim again, $k-1 \leq\left|p^{k-1} c\right|<\left|p^{k-1} a\right|$, thus $k \leq\left|p^{k-1} a\right|$. But then $a=a^{\prime}+a^{\prime \prime}\left(a^{\prime}, a^{\prime \prime} \in A_{1}\right)$, such that $a^{\prime}$ has order $p^{k-1}$ and $\left|a^{\prime \prime}\right| \geq 1$. Thus $\pi(a) \in \pi(B)+p C$ and the induction is complete.

Let us say that the Ulm invariant conditions apply to $B, A_{1}$ and $A_{2}$ if each Ulm invariant of $B$ is either finite or else the corresponding Ulm invariants of $A_{1}$ and $A_{2}$ are zero. The next lemma allows us to replace two isomorphic bounded direct summands by a common summand.

Lemma 3. Let $G=B_{1} \oplus B_{2} \oplus B^{\prime} \oplus A_{1}=C_{1} \oplus C_{2} \oplus C^{\prime} \oplus A_{2}$ such that $B_{i} \cong C_{i}(i=$ 1,2), $B_{1} \oplus B_{2} \subseteq C_{1} \oplus C_{2} \oplus A_{2}$, and $C_{1} \oplus C_{2} \subseteq B_{1} \oplus B_{2} \oplus A_{1}$. Assume that $B_{1}$ is a bounded p-group, that we are given an element $u$ of the socle of $B_{1}$, that the Ulm 
invariants of $B_{1}$ and $B_{2}$ are disjoint, and that the Ulm invariant conditions hold for $B_{1}, A_{1}$ and $A_{2}$. Then there exists $D$ such that:

(i) $G=D \oplus B_{2} \oplus B^{\prime} \oplus A_{1}=D \oplus C_{2} \oplus C^{\prime} \oplus A_{2}$;

(ii) $B_{1} \oplus B_{2} \oplus A_{1}=D \oplus B_{2} \oplus A_{1}$ and $C_{1} \oplus C_{2} \oplus A_{2}=D \oplus C_{2} \oplus A_{2}$; and

(iii) $u \in D \oplus A_{1}$.

Proof. Note that (ii) will follow from (i) and the hypothesis of the lemma if we have $D \subseteq\left(B_{1} \oplus B_{2} \oplus A_{1}\right) \cap\left(C_{1} \oplus C_{2} \oplus A_{2}\right)$.

We shall induct on the sum of the finite Ulm invariants of $B_{1}$ plus the number of infinite Ulm invariants. We shall consider decompositions $B_{1}=B_{1}^{\prime} \oplus B_{1}^{\prime \prime}$ and $C_{1}=C_{1}^{\prime} \oplus C_{1}^{\prime \prime}$ such that $B_{1}^{\prime} \cong C_{1}^{\prime}$ and $B_{1}^{\prime \prime} \cong C_{1}^{\prime \prime}$. We shall obtain $D$ as $D^{\prime} \oplus D^{\prime \prime}$.

First suppose that $B_{1}$ has an infinite Ulm invariant. Then we may take $B_{1}^{\prime}$ and $B_{1}^{\prime \prime}$ such that $B_{1}^{\prime}$ is nontrivial and has Ulm invariants disjoint from those of $B_{1}^{\prime \prime}$ (thus $\left.C_{1}^{\prime \prime}\right), A_{1}$ and $A_{2}$. Write $u=u^{\prime}+u^{\prime \prime}\left(u^{\prime} \in B_{1}^{\prime}, u^{\prime \prime} \in B_{1}^{\prime \prime}\right)$. Grouping $B_{1}^{\prime \prime}$ and $B_{2}$ with $A_{1}$ and $C_{1}^{\prime \prime}$ and $C_{2}$ with $A_{2}$, we may apply Lemma 2 with $B=B_{1}^{\prime}$ and $C=C_{1}^{\prime}$ since $C_{1}^{\prime} \subseteq B_{1} \oplus B_{2} \oplus A_{1}=B_{1}^{\prime} \oplus\left(B_{1}^{\prime \prime} \oplus B_{2} \oplus A_{1}\right)$. Thus, $G=B_{1}^{\prime}+\left(C_{1}^{\prime \prime} \oplus C_{2} \oplus C^{\prime} \oplus A_{2}\right)$. If $g \in B_{1}^{\prime} \cap\left(C_{1}^{\prime \prime} \oplus C_{2} \oplus C^{\prime} \oplus A_{2}\right)$, then $g \in C_{1}^{\prime \prime} \oplus C_{2} \oplus A_{2}$ since $B_{1} \subseteq C_{1} \oplus C_{2} \oplus A_{2}$. If $g \neq 0$, then there is an element of order $p$ in $B_{1}^{\prime} \cap\left(C_{1}^{\prime \prime} \oplus C_{2} \oplus A_{2}\right)$. The $p$-height of such an element must occur at an ordinal for which the Ulm invariants of both $B_{1}^{\prime}$ and $C_{1}^{\prime \prime} \oplus C_{2} \oplus A_{2}$ are nonzero. This contradicts the choice of $B_{1}^{\prime}$ and the assumption on Ulm invariants, thus the sum for $G$ is a direct sum. Therefore, we can take $D^{\prime}=B_{1}^{\prime}$, replacing both $B_{1}^{\prime}$ and $C_{1}^{\prime}$. Note that $u^{\prime} \in D^{\prime}$. Now group $D^{\prime}$ with $A_{1}$ and $A_{2}$ and apply induction to $B_{1}^{\prime \prime}$, replacing both $B_{1}^{\prime \prime}$ and $C_{1}^{\prime \prime}$ by $D^{\prime \prime}$, with $u^{\prime \prime} \in D^{\prime \prime}+\left(D^{\prime}+A_{1}\right)$. Put $D=D^{\prime} \oplus D^{\prime \prime}$.

If $B_{1}$ has no infinite Ulm invariant, then it is a finite group, so we may take $B_{1}^{\prime}=\langle b\rangle$ such that $u \in B_{1}^{\prime}$. Let $C_{1}^{\prime}=\langle c\rangle$. Then we have $b=m c+c^{\prime \prime}+c_{2}+a_{2}(m \in$ $\left.\mathbb{Z}, c^{\prime \prime} \in C_{1}^{\prime \prime}, c_{2} \in C_{2}, a_{2} \in A_{2}\right)$. If $p \nmid m$, we can take $D^{\prime}=B_{1}^{\prime}$. Therefore, assume that $p \mid m$. Further, $c=n b+b^{\prime \prime}+b_{2}+a_{1}\left(n \in \mathbb{Z}, b^{\prime \prime} \in B_{1}^{\prime \prime}, b_{2} \in B_{2}, a_{1} \in A_{1}\right)$ and $b_{2}=k c+\bar{c}^{\prime \prime}+\bar{c}_{2}+\bar{a}_{2}\left(k \in \mathbb{Z}, \bar{c}^{\prime \prime} \in C_{1}^{\prime \prime}, \bar{c}_{2} \in C_{2}, \bar{a}_{2} \in A_{2}\right)$. By the first equation, the order of $b_{2}$ cannot exceed the order of $c$. In the second, if $p \nmid k$, then $b_{2}$ generates a cyclic summand of $B_{2}$ of the same order as $c$, contradicting the Ulm invariants of $B_{1}$ and $B_{2}$ being disjoint. Thus $p \mid k$. Put $D^{\prime}=\left\langle b+b^{\prime \prime}+a_{1}\right\rangle$. Clearly, $G=D^{\prime} \oplus B_{1}^{\prime \prime} \oplus B_{2} \oplus B^{\prime} \oplus A_{1}$. If we can show that $G=D^{\prime}+\left(C_{1}^{\prime \prime} \oplus C_{2} \oplus C^{\prime} \oplus A_{2}\right)$, then the sum will be direct since $D^{\prime}$ and $C_{1}^{\prime}$ have the same order, which is the index of $C_{1}^{\prime \prime} \oplus C_{2} \oplus C^{\prime} \oplus A_{2}$ in $G$. It suffices to show that $c$ lies in this sum. Reading the above equations modulo $C_{1}^{\prime \prime} \oplus C_{2} \oplus C^{\prime} \oplus A_{2}$, we have $b \equiv m c, c \equiv n b+b^{\prime \prime}+b_{2}+a_{1}$, and $b_{2} \equiv k c$, hence $c \equiv\left(b+b^{\prime \prime}+a_{1}\right)+(n-1) m c+k c$. Since $p$ divides both $m$ and $k$, $c$ lies in the sum above. Now group $D^{\prime}$ with $A_{1}$ and $A_{2}$ and apply induction to $B_{1}^{\prime \prime}$ and a generator of the socle of $\left\langle b^{\prime \prime}\right\rangle$. Thus we get $D^{\prime \prime}$ which replaces $B_{1}^{\prime \prime}$ and $C_{1}^{\prime \prime}$. Taking $D=D^{\prime} \oplus D^{\prime \prime}$, and noting that $u \in D \oplus A_{1}$, the induction is finished.

Before considering countable $B$, we prove a simple extension lemma. For $\alpha$ an ordinal, we let $G^{\alpha}$ denote the $\alpha$-th Ulm subgroup of $G$.

Lemma 4. Let $G=V \oplus H$ and $G^{\alpha}=Z \oplus H^{\alpha}$. Assume that $V$ is a p-group such that $V / V^{\alpha}$ is totally projective. Then there exists $X$ such that $G=X \oplus H$ and $X^{\alpha}=Z$.

Proof. We have $G^{\alpha}=V^{\alpha} \oplus H^{\alpha}=Z \oplus H^{\alpha}$. Let $\pi$ be the projection $Z \oplus H^{\alpha} \rightarrow H^{\alpha}$ with kernel $Z$. The homomorphism $\phi: V^{\alpha} \oplus H \rightarrow H$ given by $\phi(v, h)=\pi(v)+h$ 
does not decrease $p$-heights relative to $V \oplus H=G$. Since $V^{\alpha}$ is a nice subgroup of $V$ with totally projective quotient, [2, Corollary 81.4] implies that $\phi$ extends to a homomorphism $\bar{\phi}: V \oplus H \rightarrow H$. Let $X$ be the kernel of $\bar{\phi}$. Clearly, $G=X \oplus H$. If $z \in Z$, then $z=v+h\left(v \in V^{\alpha}, h \in H^{\alpha}\right)$. Thus, $\bar{\phi}(z)=\pi(v)+h=\pi(v)+\pi(h)=$ $\pi(z)=0$, and we have $Z \subseteq X$. This implies that $Z \subseteq X^{\alpha}$, and $G^{\alpha}=X^{\alpha} \oplus H^{\alpha}=$ $Z \oplus H^{\alpha}$ shows that $Z=X^{\alpha}$.

Lemma 5. Assume that $G=B \oplus B^{\prime} \oplus A_{1}=C \oplus C^{\prime} \oplus A_{2}, B \subseteq C \oplus A_{2}, C \subseteq B \oplus A_{1}$, and that $B \cong C$ is a countable p-group. Assume the Ulm invariant conditions for $B, A_{1}$ and $A_{2}$. Then there exists $D$ such that $G=D \oplus B^{\prime} \oplus A_{1}=D \oplus C^{\prime} \oplus A_{2}, B \oplus$ $A_{1}=D \oplus A_{1}$, and $C \oplus A_{2}=D \oplus A_{2}$.

Proof. We induct on the Ulm length $\lambda$ of $B$. For $\lambda=0$, we have $B=0$ and take $D=0$. Therefore assume $\lambda>0$. As we noted in the proof of Lemma 3, we only need to show that $G=D \oplus B^{\prime} \oplus A_{1}=D \oplus C^{\prime} \oplus A_{2}$ and $D \subseteq\left(C \oplus A_{2}\right) \cap\left(B \oplus A_{1}\right)$. We will obtain $D$ by a second induction. We shall construct $B_{n}, C_{n}$ and $D_{n}$ for $n<\omega$ such that:

(a) $G=D_{n} \oplus B_{n} \oplus B^{\prime} \oplus A_{1}=D_{n} \oplus C_{n} \oplus C^{\prime} \oplus A_{2}$;

(b) $B_{n}$ and $C_{n}$ are direct summands of $B$ and $C$, respectively, $B_{n} \cong C_{n}$, and $D_{n} \subseteq D_{n+1}$

(c) $B_{n} \subseteq D_{n} \oplus C_{n} \oplus A_{2}, C_{n} \subseteq D_{n} \oplus B_{n} \oplus A_{1}$, and $D_{n} \subseteq\left(B \oplus A_{1}\right) \cap\left(C \oplus A_{2}\right)$;

(d) $B_{n}$ and $D_{n}$ have disjoint Ulm invariants; and

(e) putting $D=\bigcup_{n<\omega} D_{n}$, we have $B[p] \subseteq D \oplus B^{\prime} \oplus A_{1}$ and $C[p] \subseteq D \oplus C^{\prime} \oplus A_{2}$. Each $D_{n} \oplus B^{\prime} \oplus A_{1}$ is a direct summand of $G$, hence pure in $G$. Thus $D \oplus B^{\prime} \oplus A_{1}=$ $\bigcup_{n<\omega}\left(D_{n} \oplus B^{\prime} \oplus A_{1}\right)$ is a pure subgroup of $G$ containing the socles of $B, B^{\prime}$ and $A_{1}$, hence will equal $G$. Similarly, $G=D \oplus C^{\prime} \oplus A_{2}$. Moreover, condition (c) gives $D \subseteq\left(B \oplus A_{1}\right) \cap\left(C \oplus A_{2}\right)$, thus we will be done if we carry out the construction.

We start with $D_{0}=0, B_{0}=B$ and $C_{0}=C$. Enumerate the elements of the socles of $B$ and $C$ and alternate the construction so that each element of $B[p]$ or $C[p]$ lies in some $D_{n} \oplus B^{\prime} \oplus A_{1}$ or $D_{n} \oplus C^{\prime} \oplus A_{2}$, respectively. This will take care of condition (e). By symmetry, we may assume that $s \in B[p]$, that (a)-(d) hold (except for $D_{n} \subseteq D_{n+1}$ ), and we shall construct appropriate $B_{n+1}, C_{n+1}$, and $D_{n+1} \supseteq D_{n}$ such that $s \in D_{n+1} \oplus B^{\prime} \oplus A_{1}$.

Let $u$ be the coordinate of $s$ in $B_{n}$ in the decomposition (a). We shall achieve $s \in D_{n+1} \oplus B^{\prime} \oplus A_{1}$ if $u \in D_{n+1} \oplus B^{\prime} \oplus A_{1}$. If $u=0$, we can take $D_{n+1}=D_{n}, B_{n+1}=$ $B_{n}$ and $C_{n+1}=C_{n}$, so assume that $u \neq 0$. Thus there is an ordinal $\alpha<\lambda$ such that $u \in B_{n}^{\alpha} \backslash B_{n}^{\alpha+1}$. We may decompose $B_{n}^{\alpha}=U \oplus U^{\prime}$, where $u \in U, p^{r} U=0$, and $U^{\prime}$ has no cyclic summand of order $\leq p^{r}$. We claim that there is a decomposition $B_{n}=V \oplus B_{n+1}$ such that $V^{\alpha}=U, B_{n+1}^{\alpha}=U^{\prime}$, and for which $V$ and $B_{n+1}$ have disjoint Ulm invariants. First we use [2, Corollary 76.2] to produce countable groups $\bar{V}$ and $\bar{B}_{n+1}$ by specifying Ulm factors. By (b), $B_{n}$ is countable, thus for $\sigma<\alpha$ we may decompose the Ulm factor $\left(B_{n}\right)_{\sigma}=\bar{V}_{\sigma} \oplus\left(\bar{B}_{n+1}\right)_{\sigma}$ so that each summand is an unbounded countable direct sum of cyclic groups and the Ulm invariants are disjoint. For $\sigma \geq \alpha$, we take $\bar{V}_{\sigma}$ and $\left(\bar{B}_{n+1}\right)_{\sigma}$ to be the appropriate Ulm factors of $U$ and $U^{\prime}$, respectively. Thus we obtain countable groups $\bar{V}$ and $\bar{B}_{n+1}$ with the specified Ulm factors, hence with disjoint Ulm invariants. Moreover, [2] Corollary 77.3] implies that $\bar{V}^{\alpha} \cong U, \bar{B}_{n+1}^{\alpha} \cong U^{\prime}$, and $B_{n} \cong \bar{V} \oplus \bar{B}_{n+1}$ since we are dealing with countable groups with the same Ulm factors. Thus, we may assume that $\bar{V}^{\alpha}=U$ and $\bar{B}_{n+1}^{\alpha}=U^{\prime}$. By [2, Corollary 77.4], there is an isomorphism 
$B_{n} \cong \bar{V} \oplus \bar{B}_{n+1}$ which is the identity on $B_{n}^{\alpha}$. Using this isomorphism, we pull back $\bar{V}$ and $\bar{B}_{n+1}$ to obtain $B_{n}=V \oplus B_{n+1}$, as desired.

Since $C_{n} \cong B_{n}$, we may write $C_{n}=W \oplus C_{n+1}$, where $W \cong V$ and $C_{n+1} \cong B_{n+1}$. Thus we have $G=D_{n} \oplus V \oplus B_{n+1} \oplus B^{\prime} \oplus A_{1}=D_{n} \oplus W \oplus C_{n+1} \oplus C^{\prime} \oplus A_{2}$. Taking $\alpha$-th Ulm subgroups, $G^{\alpha}=D_{n}^{\alpha} \oplus V^{\alpha} \oplus B_{n+1}^{\alpha} \oplus\left(B^{\prime}\right)^{\alpha} \oplus A_{1}^{\alpha}=D_{n}^{\alpha} \oplus W^{\alpha} \oplus C_{n+1}^{\alpha} \oplus\left(C^{\prime}\right)^{\alpha} \oplus A_{2}^{\alpha}$. If we group $D_{n}^{\alpha}$ with $A_{1}^{\alpha}$ and $A_{2}^{\alpha}$, then (c) together with the arrangements we have made with Ulm invariants allow us to apply Lemma 3 to obtain $\mathrm{Z}$ such that:

(i) $G^{\alpha}=Z \oplus D_{n}^{\alpha} \oplus B_{n+1}^{\alpha} \oplus\left(B^{\prime}\right)^{\alpha} \oplus A_{1}^{\alpha}=Z \oplus D_{n}^{\alpha} \oplus C_{n+1}^{\alpha} \oplus\left(C^{\prime}\right)^{\alpha} \oplus A_{2}^{\alpha}$;

(ii) $V^{\alpha} \oplus D_{n}^{\alpha} \oplus B_{n+1}^{\alpha} \oplus A_{1}^{\alpha}=Z^{\alpha} \oplus D_{n}^{\alpha} \oplus B_{n+1}^{\alpha} \oplus A_{1}^{\alpha}$;

(iii) $W^{\alpha} \oplus D_{n}^{\alpha} \oplus B_{n+1}^{\alpha} \oplus A_{1}^{\alpha}=Z^{\alpha} \oplus D_{n}^{\alpha} \oplus B_{n+1}^{\alpha} \oplus A_{1}^{\alpha}$; and

(iv) $u \in Z \oplus A_{1}^{\alpha}$.

We now apply Lemma 4 to $D_{n} \oplus V \oplus B_{n+1} \oplus A_{1}$ and $D_{n} \oplus W \oplus C_{n+1} \oplus A_{2}$, utilizing (ii) and (iii) to obtain $X$ and $Y$ with $X^{\alpha}=Y^{\alpha}=Z, V \oplus D_{n} \oplus B_{n+1} \oplus A_{1}=$ $X \oplus D_{n} \oplus B_{n+1} \oplus A_{1}$, and $W \oplus D_{n} \oplus C_{n+1} \oplus A_{2}=Y \oplus D_{n} \oplus C_{n+1} \oplus A_{2}$. Since $X \cong Y \cong V$, then $X / Z \cong Y / Z \cong V / V^{\alpha}$ satisfies the Ulm invariant conditions for $X / Z, D_{n} \oplus B_{n+1} \oplus A_{1}$, and $D_{n} \oplus C_{n+1} \oplus A_{2}$. Passing to the quotient group $G / Z$, induction can be used since the Ulm length of $X / Z$ is $\alpha$. Thus we get $T \supseteq Z$ such that $G=T \oplus D_{n} \oplus B_{n+1} \oplus B^{\prime} \oplus A_{1}=T \oplus D_{n} \oplus C_{n+1} \oplus C^{\prime} \oplus A_{2}, X \oplus D_{n} \oplus B_{n+1} \oplus A_{1}=$ $T \oplus D_{n} \oplus B_{n+1} \oplus A_{1}$, and $Y \oplus D_{n} \oplus C_{n+1} \oplus A_{2}=T \oplus D_{n} \oplus C_{n+1} \oplus A_{2}$.

Put $D_{n+1}=T \oplus D_{n}$. Note that $u \in D_{n+1} \oplus A_{1}$. All requirements of the induction step from $n$ to $n+1$ are easily verified except possibly (c). For $B_{n+1}$, we have $B_{n+1} \subseteq B_{n} \subseteq D_{n} \oplus C_{n} \oplus A_{2}=D_{n} \oplus W \oplus C_{n+1} \oplus A_{2}=Y \oplus D_{n} \oplus C_{n+1} \oplus A_{2}=$ $T \oplus D_{n} \oplus C_{n+1} \oplus A_{2}=D_{n+1} \oplus C_{n+1} \oplus A_{2}$. To check that $D_{n+1} \subseteq B \oplus A_{1}$, it suffices to show that $T \subseteq B \oplus A_{1}$. But previous equalities yield $T \subseteq X \oplus D_{n} \oplus B_{n+1} \oplus A_{1}, X \subseteq$ $V \oplus D_{n} \oplus B_{n+1} \oplus A_{1}$, and $D_{n} \subseteq B \oplus A_{1}$. This completes the induction.

Proof of the Theorem. Suppose that $B=\bigoplus_{j \in J} B_{j}^{\prime}$ and $C=\bigoplus_{j \in J} C_{j}^{\prime}$, where $B_{j}^{\prime} \cong C_{j}^{\prime}$ is countable for every $j \in J$. By a standard countable back-and-forth argument for combining summands, we may assume that $B=\bigoplus_{i<\gamma} B_{i}, C=$ $\bigoplus_{i<\gamma} C_{i}, B_{i} \cong C_{i}$, and for every $\alpha<\gamma$, we have $\bigoplus_{i<\alpha} B_{i} \subseteq \bigoplus_{i<\alpha} C_{i} \oplus A_{2}$ and $\bigoplus_{i<\alpha} C_{i} \subseteq \bigoplus_{i<\alpha} B_{i} \oplus A_{1}$.

We shall construct $D_{\alpha}(\alpha \leq \gamma)$ such that $G=D_{\alpha} \oplus\left(\bigoplus_{\alpha \leq i<\gamma} B_{i}\right) \oplus A_{1}=$ $D_{\alpha} \oplus\left(\bigoplus_{\alpha \leq i<\gamma} C_{i}\right) \oplus A_{2}$, and such that for $\beta<\alpha \leq \gamma$, we have $D_{\beta} \subseteq D_{\alpha}, B_{\beta} \subseteq$ $D_{\alpha} \oplus A_{1}$, and $C_{\beta} \subseteq D_{\alpha} \oplus A_{2}$. $D_{\gamma}$ will then be the required $D$. For a limit ordinal $\alpha$, we take $D_{\alpha}=\bigcup_{\beta<\alpha} D_{\beta}$. It is clear that this will work, hence we need to consider going from $\alpha$ to $\alpha+1$. Thus we assume that

$$
\begin{aligned}
G & =D_{\alpha} \oplus B_{\alpha} \oplus\left(\bigoplus_{\alpha<i<\gamma} B_{i}\right) \oplus A_{1} \\
& =D_{\alpha} \oplus C_{\alpha} \oplus\left(\bigoplus_{\alpha<i<\gamma} C_{i}\right) \oplus A_{2},
\end{aligned}
$$

and for $\beta<\alpha$ that $B_{\beta} \subseteq D_{\alpha} \oplus A_{1}$ and $C_{\beta} \subseteq D_{\alpha} \oplus A_{2}$. We have $B_{\alpha} \subseteq \bigoplus_{i \leq \alpha} C_{i} \oplus A_{2}$ and $C_{\alpha} \subseteq \bigoplus_{i \leq \alpha} B_{i} \oplus A_{1}$, thus $B_{\alpha} \subseteq D_{\alpha} \oplus C_{\alpha} \oplus A_{2}$ and $C_{\alpha} \subseteq D_{\alpha} \oplus B_{\alpha} \oplus A_{1}$. Passing to the quotient $G / D_{\alpha}$, Lemma 5 applies to yield $D_{\alpha+1} \supseteq D_{\alpha}$ such that $G=D_{\alpha+1} \oplus\left(\bigoplus_{\alpha+1 \leq i<\gamma} B_{i}\right) \oplus A_{1}=D_{\alpha+1} \oplus\left(\bigoplus_{\alpha+1 \leq i<\gamma} C_{i}\right) \oplus A_{2}, B_{\alpha} \subseteq D_{\alpha+1} \oplus A_{1}$, and $C_{\alpha} \subseteq D_{\alpha+1} \oplus \bar{A}_{2}$. The Theorem is proved. 


\section{REFERENCES}

[1] P. Crawley, The cancellation of torsion abelian groups in direct sums, J. Algebra 2 (1965) 432-442. MR 32:5732

[2] L. Fuchs, Infinite Abelian Groups, Vol. I, Vol. II, Academic Press, New York 1970, 1973. MR 41:333, MR 50:2362

[3] R. Göbel, W. May, Modular group algebras of $\aleph_{1}$-separable p-groups, to appear.

Fachbereich 6, Mathematik und Informatik, Universität Essen, Universitätsstr. 3, 45117 Essen, Germany

E-mail address: R.Goebel@Uni-Essen.De

Department of Mathematics, University of Arizona, Tucson, Arizona 85721

E-mail address: may@math.arizona.edu 